\title{
Stability Analysis of the Mode-Locking Dynamics in a Laser Cavity with a Passive Polarizer
}

\author{
Edwin Ding ${ }^{1}$ and J. Nathan Kutz ${ }^{2}$
}

\begin{abstract}
A low-dimensional model is constructed via a variational formulation which characterizes the mode-locking dynamics in a laser cavity with a passive polarizer. The theoretical model accounts explicitly for the effects of the passive polarizer with a Jones matrix. In combination with the nonlinear interaction of the orthogonally polarized electromagnetic fields, the evolution of the mode-locked state reduces to the nonlinear interaction of the amplitude, width and phase chirp. This model allows for an explicit analytic prediction of the steady-state mode-locked state (fixed point) and its corresponding stability. The stability analysis requires a center manifold reduction which reveals that the solution decays to the mode-locked state on a timescale dependent on the gain bandwidth and the net cavity gain. Quantitative and qualitative agreement is achieved between the full governing model and the low-dimensional model, thus providing for an excellent design tool for characterizing and optimizing mode-locking performance.
\end{abstract}

Keywords: mode-locking, variational reduction, passive polarizer, Jones matrix, gain saturation

\section{Introduction}

It has been approximately two decades since it was first established experimentally that stable and robust modelocking could be achieved using a passive polarizer as an effective saturable absorber in a laser cavity [1]. The development of such sources of ultrashort light pulses has had major scientific impact in that it has enabled direct observation of ultra-fast processes in nature and studies of matter under extreme conditions. The generation of ultrashort light pulses has been the critical technology in driving these ultrafast science studies. A mode-locked laser cavity with a passive polarizer is a prime example of a technologically promising and commercially developed ultrafast device. And despite its wide-spread commercial and academic usage, the underlying theory quantifying the mode-locking stability and dynamics remains incomplete. In this manuscript, we further advance the theoretical understanding of the mode-locking dynamics with a passive polarizer by first demonstrating that the laser

\footnotetext{
*Department of Applied Mathematics, University of Washington, Seattle, WA 98195-2420, U.S.A., Tel/Fax: 1 206-685-9395/1440, 2 206-685-3029/1440, Emails: 1 ding@amath.washington.edu, ${ }^{2}$ kutz@amath.washington.edu
}

cavity can be accurately represented by a low-dimensional model that captures the nonlinear amplitude, width and phase chirp dynamics, and second, explicitly calculating the stability of the mode-locked solutions and their global-attracting nature within the context of the lowdimensional model. The model can also easily capture the full intracavity pulse fluctuations which are critical in driving the underlying mode-locking dynamics. It is to our knowledge, the first analytic calculation of the stability of the mode-locked solution in a model which explicitly accounts for the polarization dynamics and which includes the decay rate to the attracting mode-locked state.

A common feature to all mode-locked lasers is the intensity discrimination which is achieved by the mode-locking mechanism [1,2]. Such intensity discrimination, which can be also thought of as saturable absorption, is the underlying mechanism responsible for Kerr lens modelocking. The focus here will be strictly on the intensity discrimination achieved in a cavity with intra-cavity polarization rotation in conjunction with a passive polarizer (see figure 1). Incorporation of the full nonlinear polarization dynamics is hard to handle from a theoretical standpoint. Certainly, there are no difficulties in simulating the full system with polarization rotation $[3,4]$, but extracting analytic results remains a mathematically challenging proposition. This has led to the consideration of reduction techniques which allow for simplification of the governing equations while retaining the key polarization rotation elements. However, unlike previous work [5], the gain saturation dynamics and full Jones matrix for a non-ideal polarizer is included in our analysis here. This not only gives a more physically meaningful model, but it also allows for an explicit calculation of the decay rate (global attraction) to the mode-locked solution. Thus the present work greatly extends the theoretical framework and fundamental understanding of the laser cavity with a passive polarizer and further circumvents the analytically intractable results of previous work.

\section{Governing Equations}

To accurately quantify the evolution of the electromagnetic field, the dominant physical effects in the laser cavity must be included. Specifically, the leading order dynamics must include the effect of chromatic dispersion, self-phase modulation, cross-phase modulation for the or- 


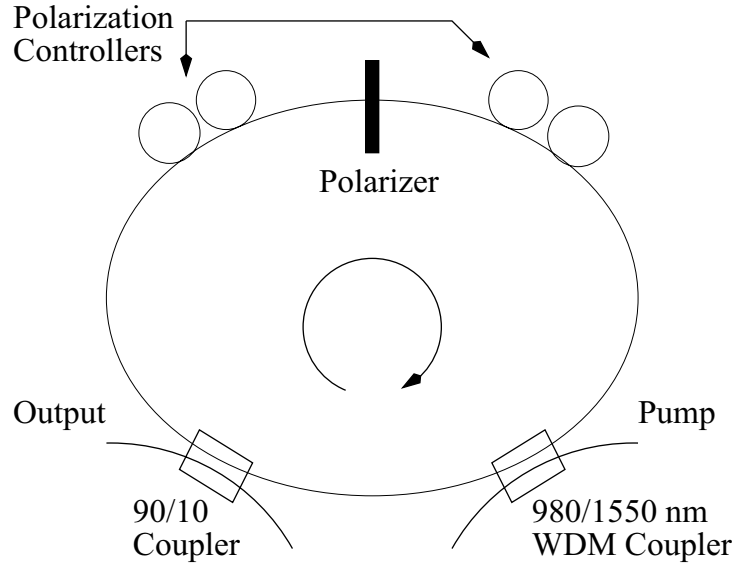

Figure 1: Experimental configuration of a ring laser cavity that includes a passive polarizer, Erbium-doped amplification, output coupler and polarization ears. The polarization ears can be used to tune the cavity birefringence. For a short cavity, the polarizer suppresses the polarization rotation and phase-slip generated from the birefringence so that stable mode-locking is achieved.

thogonal components of the polarization vector in the fast- and slow-fields, gain saturation, cavity attenuation and the Jones matrix rotation model for the passive polarizer. A passive polarizer is applied periodically in the governing equations since its effects are experienced once per round trip in the cavity. These effects are a critical component determining the mode-locking dynamics and stability. Figure ?? illustrates a basic experimentally realizable laser cavity configuration. Such an experimental configuration has been the subject of experimental, computational and theoretical investigations for approximately two decades.

\subsection{Intra-cavity fiber propagation}

We start by considering the propagation of the electromagnetic field in a birefringent optical fiber. The intracavity evolution of the slowly varying envelope of the electric field in an optical fiber subjected to chromatic dispersion, Kerr nonlinearity, polarization effects, attenuation, and bandwidth limited gain is given by the system of coupled nonlinear Schrödinger equations (CNLSs):

$i \frac{\partial U}{\partial Z}+\frac{1}{2} \frac{\partial^{2} U}{\partial T^{2}}-K U+\left(|U|^{2}+A|V|^{2}\right) U+B V^{2} U^{*}=i R_{U}$ (1a)

$i \frac{\partial V}{\partial Z}+\frac{1}{2} \frac{\partial^{2} V}{\partial T^{2}}+K V+\left(A|U|^{2}+|V|^{2}\right) V+B U^{2} V^{*}=i R_{V}$

where:

$$
R_{\sigma}=G(Z)\left(1+\tau \frac{\partial^{2}}{\partial T^{2}}\right) \sigma-\gamma \sigma
$$

and

$$
G(Z)=\frac{2 g_{0}}{1+\int_{-\infty}^{\infty}\left(|U|^{2}+|V|^{2}\right) d T}
$$

Here $G(z)$ models the saturating gain dynamics $[1,2]$ with bandwidth $\tau$. The gain (pumping) strength and loss of the fiber are represented by the parameters $2 g_{0}$ and $\gamma$ respectively. They are both non-negative constants where $2 g_{0}>\gamma$ in order for cavity net-gain to exist and modelocking to occur. This system models linearly polarized light propagating in a birefringent optical fiber in nondimensionalized form for which the $U$ and $V$ fields are orthogonally polarized components of the electric field. The birefringence strength parameter, $K$, determines the relative phase velocity difference between the $U$ and $V$ fields. The material properties of the optical fiber determine the values of nonlinear coupling parameters $A$ and $B$. These parameters satisfy $A+B=1$ by axisymmetry and, for the physical system considered here, take on the specific values $A=2 / 3$ and $B=1 / 3$.

\subsection{Polarization dynamics}

In addition to the cavity propagation effects, a linear polarizer is added to the cavity to filter the polarization state of the mode-locked pulse every roundtrip. In principle, the mode-locking works as follows: a propagating pulse experiences an intensity dependent polarization rotation as governed by (1). Upon propagating over the length of the cavity, the pulse is then subjected to the polarization filtering effect generated from the linear polarizer. Specifically, only the polarization state aligned with the linear polarizer's principle axis is not attenuated. Thus the intensity-dependent rotation in conjunction with the linear polarizer act to select a specific pulse intensity, i.e. the mode-locked pulse solution.

In the ideal case, the pulse coming out from the polarizer will be linearly polarized so that the phase difference between the orthogonal polarization components $U$ and $V$ will be exactly zero. For a non-ideal polarizer, there will be some leakage of the orthogonal component, and this can be modeled with a Jones matrix [6]:

$$
\left(\begin{array}{c}
U^{+} \\
V^{+}
\end{array}\right)=\left(\begin{array}{cc}
c^{2}+\alpha s^{2} & (1-\alpha) s c \\
(1-\alpha) s c & \alpha c^{2}+s^{2}
\end{array}\right)\left(\begin{array}{c}
U^{-} \\
V^{-}
\end{array}\right)
$$

Here the superscript \pm denotes the state of the components before $(-)$ and after $(+)$ passage through the polarizer. The parameters $c=\cos \theta$ and $s=\sin \theta$ where $\theta$ is the angle between the cavity fiber's fast axis and the polarizer's principle axis. The parameter $\alpha$ is a constant between 0 and 1 that measures the imperfectness (leakage) of the polarizer ( $\alpha=0$ represents an ideal polarizer). For $\alpha=1$, the Jones matrix reduces to the identity matrix so that the polarizer produces no effect upon the pulse propagation.

Figure 2 demonstrates the ideal mode-locking behavior expected in the cavity. This clearly shows that the initial white-noise condition settles to the ideal mode-locked solution on a timescale of thousands of round trips. The 

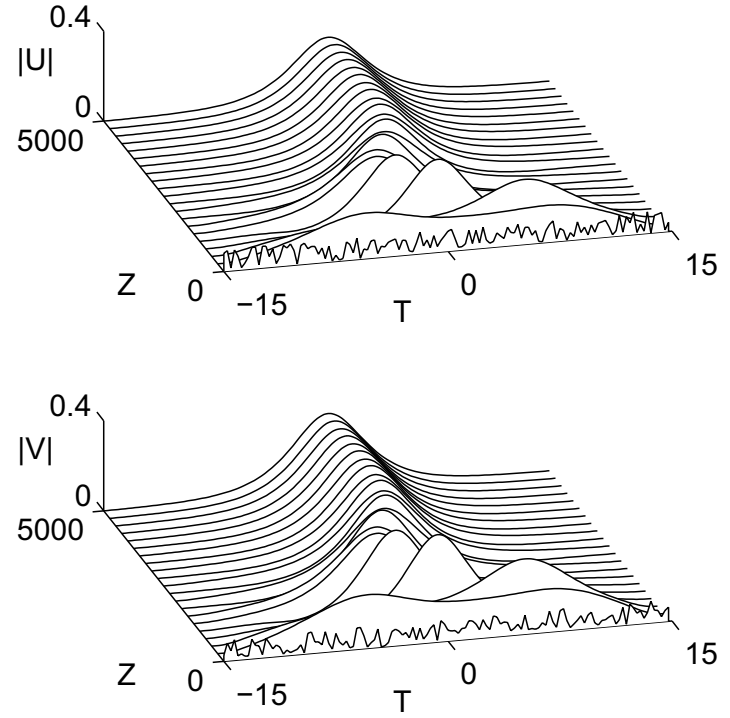

Figure 2: Ideal mode-locking behavior in the laser cavity with a passive polarizer. Here, the white-noise initial condition settles to the attracting mode-locked solution on the order of thousands of round trips. For this computation, $g_{0}=0.1, \tau=0.1, \gamma=0.1, K=1$ and $\alpha=0.01$ with the polarizer set so that $\theta=0.28 \pi$.

well-known and often studied mode-locking behavior depicted in these two figures persists over a wide range of parameter space. Indeed, its robustness to changes in the parameters are what make the mode-locked laser with a passive polarizer such a promising commercial device. The simplicity of the solution form that develops in the mode-locking process is what makes the laser cavity amenable to a low-dimensional reduction via the variational method. It is the low-dimensional model that is the focus of this manuscript.

\section{Low-Dimensional Dynamics}

The CNLS system (1) with (4) doesn't admit exact solution and therefore analysis remains a mathematically challenging proposition. A low-dimensional model is constructed via a variational formulation. This is a common practice in a wide variety of engineering, physical, and biological systems and applications. The general procedure used here was first used by Bondeson et al. [7] in the context of soliton perturbation theory.

\subsection{Variational reduction}

Fundamental to the variational reduction considered here is the selection of a specific solution form. Consistent with numerical and experimental observations, we as- sume an ansatz of the form:

$$
\left(\begin{array}{l}
U \\
V
\end{array}\right)=\left(\begin{array}{c}
\exp (-i \psi / 2) \cos P \\
\exp (+i \psi / 2) \sin P
\end{array}\right) \sqrt{\eta} \operatorname{sech} \omega T \exp \left[i\left(\beta T^{2}+\phi / 2\right)\right]
$$

where the parameters $\eta, \omega, \beta, P, \psi$ and $\phi$ are functions of $Z$ representing the amplitude, pulse width, quadratic chirp, polarizaion angle, phase difference and absolute phase respectively. The generalized Euler-Lagrange equation of the CNLS system (1) gives the approximate intracavity evolution of each of these parameters [7, 8, 9]. The ability of the generalized Euler-Lagrange equation to incorporate the dissipative (non-Hamiltonian) terms $R_{U}$ and $R_{V}$ is critical since dissipation often drives the stability and dynamics of a given system. The absolute phase $\phi$ is not coupled to any of the above equations and hence its evolution is neglected. Thus the original, infinite-dimensional evolution equation (1) reduces to a $5 \times 5$ nonlinear system of differential equations governing the amplitude, width, chirp, polarization and phase-slip.

\subsection{Locked Polarizaton and Phase-Slip}

Although a low-dimensional description of the governing evolution equations (1) has been constructed, the periodic polarizer dynamics and filtering remains to be applied. The periodic application of polarizer, which is modeled by the Jones matrix (4), induces discrete jumps in the amplitude $(\eta)$, polarization $(P)$ and phase-slip $(\psi)$ after every round-trip. This discrete nature of the problem makes stability calculations difficult. However a fundamental feature associated with the polarizer can be identified from numerical simulations of the $5 \times 5$ lowdimensional model: the polarizer controls the polarization state and the phase-slip between the $U$ - and $V$ - fields of the pulse. Specifically, the periodic application of the polarizer (4) maintains the polarization state near the set value of $\theta(P(Z) \approx \theta)$. Furthermore, the phase-slip is reset to nearly zero $(\psi(Z) \approx 0)$ for a nearly ideal polarizer $(\alpha \ll 1)$ upon passage through the polarizer according to the Jones matrix (4). It is also noticed that the jump in amplitude becomes insignificant $\left(\eta^{+} \approx \eta^{-}\right)$after tens of round-trips. Thus as a leading-order approximation, we can neglect the evolutions of polarization state $P$ and phase-slip $\psi$, as well as the discrete jumps in the amplitude $\eta$. By incorporating the effects of the polarizer this way, the discrete system (due to the periodic application of the polarizer) can be approximated by a continuous system of three differential equations. Essentially, such an approximation averages into the governing equations the polarization dynamics. The resulting $3 \times 3$ system of 


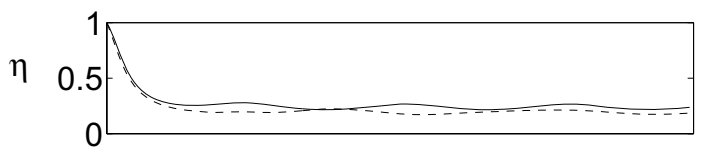

$\omega$
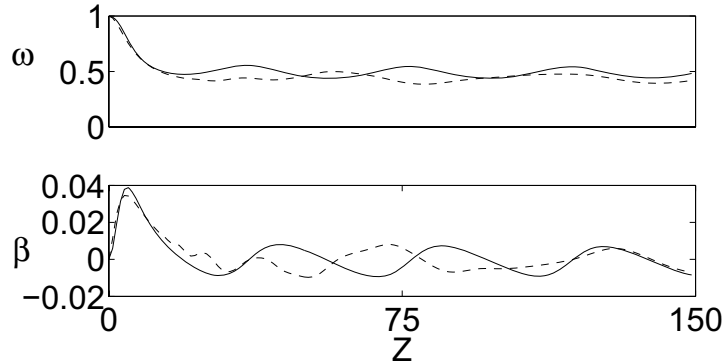

Figure 3: Comparison of the full governing evolution model (1) and (4) (dotted lines) with the three-degree of freedom low-dimensional reduction (6) (solid lines). The parameters are same as those used in figure 2 . The mode-locking dynamics in the laser cavity is accurately captured with the three-degree of freedom system with the primary difference being in a phase-slip that develops due to the slightly different periods of oscillation in the two systems. Regardless, all the critical features of the mode-locking process, including the oscillatory decay and its associated timescales, are captured very well with the low-dimensional model considered.

nonlinear equations is then given by

$$
\begin{aligned}
& \frac{d \eta}{d Z}=\frac{1}{15 \pi^{2} \omega(2 \eta+\omega)} \times\left[2 \eta \left(6 g_{0} \tau \pi^{4} \beta^{2}-30 \pi^{2}(\gamma+\beta) \eta \omega\right.\right. \\
& \left.+15 \pi^{2}\left(2 g_{0}-\gamma-\beta\right) \omega^{2}-10 g_{0} \tau\left(12+\pi^{2}\right) \omega^{4}\right] \\
& \frac{d \omega}{d Z}=\frac{32 \pi^{2} g_{0} \tau \beta^{2}-240 g_{0} \tau \omega^{4}-30 \pi^{2} \beta \omega(2 \eta+\omega)}{15 \pi^{2}(2 \eta+\omega)} \\
& \frac{d \beta}{d Z}=\frac{6(2 \eta+\omega)\left[\omega^{2}\left(\omega^{2}-\eta\right)-\pi^{2} \beta^{2}\right]-8\left(3+\pi^{2}\right) g_{0} \tau \beta \omega^{3}}{3 \pi^{2}(2 \eta+\omega)}
\end{aligned}
$$

The direct comparison of this low-dimensional model and the full model (1) shows them to be qualitatively similar with very good quantitative agreement in the amplitude, width and chirp of the achieved mode-locked solution (see figure 3). The primary difference between the models is that the oscillation period of the fast scale dynamics is slightly different between the models, creating a phase-slip between the quantities of interest. However, this does not impact the quality and usefulness of the low-dimensional (now a $3 \times 3$ nonlinear system) approximation. Figure 4 demonstrates the excellent agreement between the corresponding steady-state solutions for both the CNLS and $3 \times 3$ systems.

\section{Results}

The reduction of the laser cavity dynamics from an infinite-dimensional system (1) with a periodic pertur-
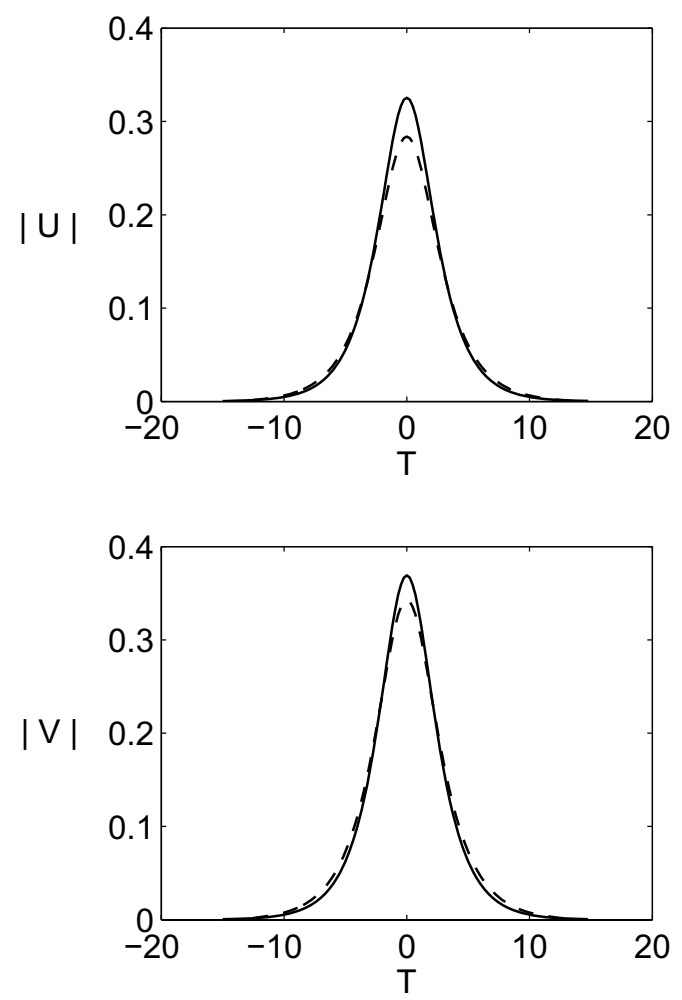

Figure 4: Comparison of the steady-state mode-locked solution for the full governing evolution model (1) and (4) (dotted lines) with the three-degree of freedom low-dimensional reduction (6) (solid lines). The steady-state mode-locked solution of the $3 \times 3$ model is achieved (after several thousand round trips) for $(\eta, \omega, \beta)=\left(0.2421,0.4921,-90 \times 10^{-4}\right)$, which should be compared to the full evolution values of $(\eta, \omega, \beta)=$ $\left(0.1982,0.4461,-14 \times 10^{-4}\right)$ with parameter values same as those used in figure 2 . Thus the $3 \times 3$ system reproduces the pulsewidth and height to within $\approx 10 \%$ and $\approx 20 \%$ respectively. The comparison between the transient responses of both models is given in figure 3 .

bation (4) to a nonlinear, three-degree of freedom of system (6) allows for significant analytic progress to be made. This variational reduction specifically allows for a complete characterization of the stability dynamics and an evaluation of the timescales necessary for the cavity to mode-lock. Highlighted in the analytic calculations is the dependence of the mode-locking stability on key physical parameters in the laser cavity. In fact the modelocked pulse is a fixed point of the reduced system (6). It is shown that this fixed point only depends on $g_{0}, \tau$ and $\gamma$, thus the same mode-locked state is achieved once these parameters are fixed. Stability of the mode-locked state is determined by performing a standard phase-plane analysis of (6) around the fixed point. Without the fiber loss and bandwidth-limited gain, the leading order system exhibits oscillatory behavior, which agrees with previous 

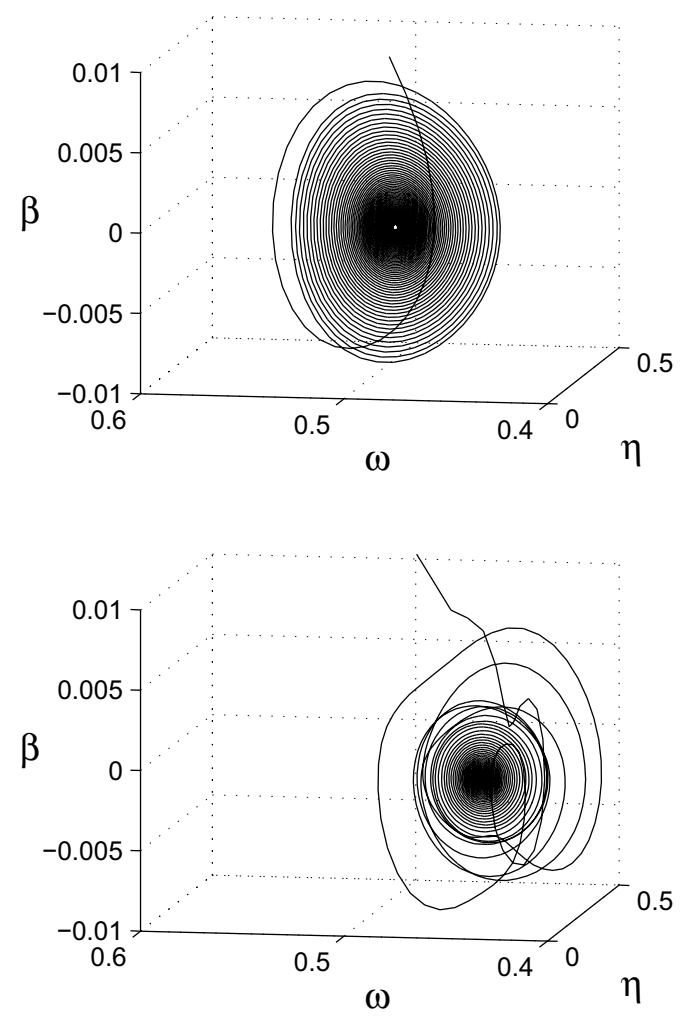

Figure 5: Three-dimensional phase-plane evolution for the low-dimensional model (6) (top) and the full governing equations (1) with (4) (bottom). The threedimensional approximation to the evolution dynamics is remarkably good as the fixed point and oscillation period of the decay is quantitatively close to the full evolution equation aside from an initial transient behavior in the full equations.

work [5]. The presence of dissipative terms perturbs the eigenvalues associated with the linearization and generates terms with slightly negative real parts, but at different orders of magnitude. Such a calculation is essentially a center manifold reduction as it calculates the higherorder corrections to the leading-order oscillatory modes. It is also shown that the decay rates (eigenvalues) of different modes depend primarily on two quantities: the bandwidth limit parameter $\tau$ and the amount of net cavity gain $2 g_{0}-\gamma$. The larger these quantities, the more quickly mode-locking occurs. This shows that the fundamental stability is driven by the gain/loss perturbation. Figure 5 illustrates the mode-locking behavior for both the full system (1) with (4) and the low-dimensional model (6). In both cases the dynamics settles to the twodimensional center manifold. This center manifold is expected as the leading-order behavior is an oscillatory dynamics. The decay time to the fixed point (mode-locked solution) is determined by the eigenvalues with negative real parts.
The above results are independent of the orientation $\theta$ of the polarizer provided that the reduction of the $5 \times 5$ system to the $3 \times 3$ system (6) is justified. In this case mode-locking can be achieved regardless of the polarizer's orientation relative to the fast- and slow-axis of the fiber. This is a much stronger stability statement than the one obtained in [5], where mode-locking is only possible when $\theta \approx 0$ or $\pi / 2$. Note that if the cavity length is very long, or if the birefringence parameter $K$ is large, the polarizer may not be efficient to control the polarization state $P$ and to suppress the phase-slip $\psi$. In this case, a full treatment of the original $5 \times 5$ model would need to be considered.

\section{Conclusion}

This work advances the theoretical understanding of the laser cavity by constructing a low-dimensional description of the underlying dynamics. Indeed, it is shown that a $3 \times 3$ continuous system of differential equations modeling the amplitude $(\eta)$, width $(\omega)$ and chirp $(\beta)$ fluctuations is adequate to capture both qualitatively and quantitatively the mode-locking behavior. Such a tremendous reduction in the system parameters allows for a complete analytic characterization of the stability dynamics. It is shown that the resulting mode-locked pulse is a fixed point of the reduced system. This fixed point is at leading order, orbitally stable. A center manifold analysis constructs the higher-order corrections and shows that the system reduces to a two-dimensional stable manifold whose oscillations period is determined by the net cavity gain $2 g_{0}-\gamma$ and whose decay time to the mode-locked state is dependent primarily upon the gain bandwidth and the net cavity gain. Quantitative agreement (within $\approx 20 \%$ ) is achieved with the full governing system, validating the explicit analytic formulas for the decay rate and oscillation period. This is, to our knowledge, the first set of analytic calculations for the mode-locking stability as well as the first low-dimensional model to explicitly include the loss and bandwidth-limited gain dynamics.

Although the results are restricted to the case of anomalous dispersion, the technique we used provides excellent framework for exploring mode-locking behavior in fibers operating at the normal dispersion regime $[10,11]$, which is modeled by the cubic-quintic complex GinzburgLandau equation whose coefficients are related to the polarization settings of the cavity $[12,13]$.

\section{References}

[1] H. A. Haus, "Mode-Locking of Lasers," IEEE J. Sel. Top. Quant. Elec., V6, N6, pp. 1173-1185, 11/00.

[2] J.N. Kutz, "Mode-locked Soliton Lasers," SIAM Rev., V48, N4, pp. 629-678, 11/06. 
[3] D. Y. Tang, W. S. Man and H. Y. Tam, "Stimulated soliton pulse formation and its mechanism in a passively mode-locked fibre soliton laser," Opt. Comm., V165, N4-6, pp. 189-194, 7/99.

[4] D. Y. Tang, W. S. Man, H. Y. Tam and P. Drummond, "Observation of bound states of solitons in a passively mode-locked fibre soliton laser, Phys. Rev. A, V64, 033814, 2001.

[5] A. Kim, J. N. Kutz, and D. Muraki, "Passive modelocking in optical fiber lasers by nonlinear polarization rotation," IEEE J. Quant. Elec., V36, N4, pp. 465-471, 4/00.

[6] A. Gerrard, J. M. Burch, Introduction to matrix methods in optics, Dover, 1994.

[7] A. Bondeson, M. Lisak and D. Anderson, "Soliton Perturbations: A Variational Principle for the Soliton Parameters," Physica Scripta., V20, pp. 479-485, 1979 .

[8] D. Anderson, M. Lisak and A. Berntson, "A variational approach to nonlinear evolution equations in optics," Pramana J. of Phys., V57, N5\&6 pp. 917$936,11 / 01$.

[9] M. Midrio, S. Wabnitz, P. Franco, "Perturbation theory for coupled nonlinear Schrödinger equations," Phys. Rev. E, V54, N5 pp. 5743-5751, 11/96.

[10] B. G. Bale and J. N. Kutz, "Variational method for mode-locked lasers," J. Opt. Soc. Am. B, V25, N7, pp. 1193-1202, 7/08.

[11] A. Chong, W. Renninger, F. Wise, "Properties of normal-dispersion femtosecond fiber lasers," J. Opt. Soc. Am. B, V25, N2, pp. 140-148, 2/08.

[12] W. Renninger, A. Chong, F. Wise, "Dissipative solitons in normal-dispersion fiber lasers," Phys. Rev. A, V77, 023814, 2008.

[13] A. Komarov, H. Leblond, F. Sanchez, "Quintic complex Ginzburg-Landau model for ring fiber lasers," Phys. Rev. E, V72, 025604, 2005. 